\section{Adenovirus E1A and Ewing tumors}

In the December 1999 issue of Nature Medicine, Melot and Delattre ${ }^{1}$ and Kovar ${ }^{2}$, challenged the Sanchez-Prieto et al. September 1999 article $^{3}$ proposing that the adenoviral E1A gene induces the Ewing tumor fusion transcript EWS-FLI1. Here Sanchez-Prieto et al. respond...Ed.

Sanchez-Prieto et al. reply-Both letters question the association between E1A and EWS-FLI1 by studying HEK293 cells using RT-PCR. HEK293 cells derive from human embryonic kidney cells immortalized by adenoviruses many years ago ${ }^{4}$. Since then, several different cell subclones of 293 and derived cells have been generated (HEK293, 293-T, Phoenix and Bosc23, for example). In our experiments, we studied HEK293 cells (obtained directly from the American Type Culture Collection (ATCC)), HEK293-T cells (obtained from S. Gutkind, National Institutes of Health), HEK293 cells cultured for a long time in our laboratory, and the Phoenix and BOSC23 cells. We were able to detect fusion transcripts of types 1 and 2 (always with nested PCR) in some subclones of 293 cells: type 2 in our HEK293 cells, type 1 in the HEK293 from the ATCC, and an almost-undetectable fusion transcript in the HEK293-T. The results were variable and depended on many parameters, including PCR conditions, RNA extraction, the primer used to generate cDNA (FLI1-specific was better than random) and the cell lines studied. We detected fusion transcripts much more efficiently in the Bosc23 and Phoenix cells. Therefore, in response to Melot and Delattre and Kovar, we can only say that detection of fusion transcript EWS-FLI1 in HEK293 cells may depend on the subclones studied and assay conditions, and that an association between a high level of E1A protein and the EWS-FLI1 fusion product is not always found.

We agree with Melot and Delattre that the different sizes of EWS-FLI1 proteins and cDNAs may be associated with different retroviral infections in IMR90 cells. We have detected fusion transcripts of types 1 and 2 in HEK293 and derived cells. In any case, the 293 and derived cells are aneuploid and polyclonal, and we interpret this to mean that the different sizes of the EWS-FLI1 ferent subclones of 293 cells. fusion transcripts can be related to dif-

Melot and Delattre also questioned our interpretation of our fluorescent in situ hybridization (FISH) results using a cosG9 chromosome 22 cosmid (Fig. $3 b$ in ref. 3). In fact, we incorrectly labeled this figure, which actually shows FISH with a centromeric probe of chromosome 11 and the Vysis LDI DiGeorge/Dual Color Probe, but not cosG9. Our original study used several probes for FISH, including those that recognize whole chromosomes 11 and 22 , centromeric probes, $\cos \mathrm{G} 9, \cos 1 \mathrm{~d} 1$ and the DiGeorge probe. This last probe contains a mixture of TUPLE1 (22q11.2) in orange and LSI AASA (22q13) in green. In cells with the translocation, we detected the probe marked in green (terminal region of chromosome 22) and the centromeric probe of chromosome 11 (Fig. $3 b$ in ref. $3)$. We apologize for this mistake.

In our paper, we emphasized that both IMR90 and HeLa E1A-expressing cells contain the fusion transcript EWS-FLI1 in a substantial number of cells. We have confirmed these results in new experiments (always after selection of E1A-expressing cells). Kovar mentions that they have studied the HeLa cell line 70 hours after transient transfection, with a transfection efficiency of only $2 \%$ of the cells. We think that the EWS-FLI1 fusion product is unlikely to be detected in those conditions.

Kovar did not find E1A sequences in any of either the 27 Ewing tumor cell lines or the 19 primary Ewing tumors he examined. We have subsequently studied three Ewing tumor cell lines (RD-ES, CADO and MHH-ES-1) and tumors from 32 patients with Ewing tumors (14 with EWS-FLI1 type 1 fusion, 15 with EWS-FLI1 non-type 1 fusion and 3 with EWS-ERG fusion), normal tissue of these patients, and other tumors obtained at similar ages. Using PCR, RT-PCR, Southern blot analysis and sequencing, we have detected E1A sequences in all the cell lines studied and in 14 of the 32 (43\%) Ewing tumor samples, representing both of the fusion types (data not shown.)

The presence of the EWS-FLI1 fusion could be just one of the myriad effects (direct or indirect) of adenovirus E1A in human cells ${ }^{3,5}$. As yet, we cannot conclude that EWS-FLI1 expression is necessary or important for the transforming properties of E1A. Nor do we know how adenovirus E1A induces the fusion, nor can we discount the possibility that that E1A mediates a selective advantage for EWS-FLI1 expressing cells arising spontaneously ${ }^{6}$. The finding that a substantial number of Ewing tumors contain E1A sequences strengthens the association between E1A, EWS-FLI1 and Ewing tumors.

E. DE Alava ${ }^{1}$, R. SANChez-Prieto ${ }^{2} \&$ S. RAMON Y CAJAL ${ }^{2}$

'Clínica Universitaria de Navarra

Avda Pío XII 36

31008 Pamplona, Spain

${ }^{2}$ Clínica Puerta de Hierro, c/San Martín de Porres

4, 28035 Madrid, Spain

1. Melot, T. \& Delattre, O. E1A and the Ewing tumor translocation. Nature Med. 5, 1331 (1999).

2. Kovar, H. E1A and the Ewing tumor translocation. Nature Med. 5, 1331 (1999).

3. Sanchez-Prieto, R. et al. An association between viral genes and human oncogenic alterations: The adenovirus E1A induces the Ewing tumor fusion transcript EWS-FLI1. Nature Med. 5, 1076-1081 (1999).

4. Graham, F.L., Smiley, J., Russell, W.C. \& Nairn, R. Characteristics of a human cell line transformed by DNA from human adenovirus type 5. J. Gen. Virol. 36, 59-72 (1977).

5. Caporossi, D. \& Bacchetti, S. Definition of adenovirus type 5 functions involved in the induction of chromosomal aberrations in human cells. I. Gen. Virol. 71, 801-808 (1990).

6. Kirn, D. \& Hermiston, T. Induction of an oncogenic fusion protein by a viral gene-a new chapter in an old story. Nature Med. 5, 991-992 (1999).

\section{A new Editor for Nature Medicine}

The Publishers are very pleased to announce a new Editor for Nature Medicine, Dr. Beatrice Renault.

After completing her Ph.D. and postdoctoral research (University of Pavia, Italy; University of Leiden, The Netherlands and Albert Einstein College of Medicine, US), Beatrice joined Nature's London-based editorial group where she had responsibility for genetics, and molecular neurobiology. After a year with Nature, Beatrice moved to New York to take up the position of Senior Editor, Nature Medicine, with overall responsibility for the research content of the journal.

The out going Editor, Dr. Adrian J. Ivinson, has been appointed Publisher for Nature's monthly journals. 